\title{
Tumor-associated macrophages induce the expression of FOXQ1 to promote epithelial-mesenchymal transition and metastasis in gastric cancer cells
}

\author{
JIAN GUO $^{1 *}$, YAN YAN ${ }^{2 *}$, YU YAN $^{3 *}$, QINYUE GUO $^{4}$, MINGXIN ZHANG $^{5}$, \\ JIA ZHANG $^{2}$ and DAVID GOLTZMAN ${ }^{6}$
}

\begin{abstract}
${ }^{1}$ Department of Hepatobiliary Surgery, ${ }^{2}$ The Second Department of Thoracic Surgery, Departments of ${ }^{3}$ Breast Surgery and ${ }^{4}$ Critical Care Medicine, The First Affiliated Hospital of Xi'an Jiaotong University, Xi'an, Shaanxi 710061; ${ }^{5}$ Department of Gastroenterology, Tangdu Hospital, Fourth Military Medical University, Xi'an, Shaanxi 710038, P.R. China;

${ }^{6}$ Departments of Medicine and Physiology, McGill University, Montreal, Quebec H3A 1A1, Canada
\end{abstract}

Received February 21, 2017; Accepted July 27, 2017

DOI: $10.3892 /$ or.2017.5877

\begin{abstract}
Gastric cancer (GC) is one of the most common malignancies, and is the second leading cause of cancerrelated deaths worldwide. Macrophages infiltrated in the tumor microenvironment (TME) called tumor-associated macrophages (TAMs) are key orchestrators in TME. In GC, it has been reported that infiltration of TAMs is associated with epithelial-mesenchymal transition (EMT)-related proteins in human GC tissues, but the exactly mechanism has not been clarified. In the present study, we aimed to elucidate the underlying mechanism of TAMs on GC cells. THP-1 cells were used to investigate the effects of TAMs on GC cells. The effects of invasion and migration induced by coculture with TAMs were investigated by Transwell invasion and wound healing assays. The expression of EMT-related genes and forkhead box Q1 (FOXQ1) were examined in MKN45 and MKN74 cells after being co-cultured with TAMs. The density of TAMs and the expression of FOXQ1 were analyzed by immunohistochemistry in GC tissues. Our results revealed that, co-culture with TAMs promoted the invasion and migration of GC cells. Co-culture with TAMs induced EMT in GC cells. FOXQ1 is essential for TAM-induced EMT and metastasis in GC cells. Furthermore,
\end{abstract}

Correspondence to: Dr Mingxin Zhang, Department of Gastroenterology, Tangdu Hospital, Fourth Military Medical University, 1 Xinsi Road, Xi'an, Shaanxi 710038, P.R. China

E-mail: zmx3115@163.com

Dr Jia Zhang, The Second Department of Thoracic Surgery, The First Affiliated Hospital of Xi'an Jiaotong University, 277 West Yanta Road, Xi'an, Shaanxi 710061, P.R. China

E-mail: zhangjiaxjtu@163.com

${ }^{*}$ Contributed equally

Key words: TAMs, epithelial-to-mesenchymal transition, invasion, migration, FOXQ1 silencing of FOXQ1 blocked the effect of TAM-enhanced EMT and metastasis of GC cells. High expression of CD68 was correlated with positive FOXQ1 expression ( $\mathrm{r}=0.613$; $\mathrm{P}<0.001)$ in clinical GC samples. Our data provided evidence that TAMs promote EMT, invasion and migration of GC cells via FOXQ1. Therefore, the TAM/FOXQ1 axis may represent a novel target for GC cells.

\section{Introduction}

Gastric cancer (GC) is one of the most common malignancies, and is the second leading cause of cancer-related death worldwide (1). Although rapid advancements in chemotherapy, radiation therapy, and gastric resection are widely used for GC patients, the 5-year overall survival rate has remained at $28 \%$ owing to tumor metastasis $(2,3)$.

The tumor microenvironment (TME) is comprised of tumor cells, tumor stroma, blood vessels, infiltrating inflammatory cells and a variety of associated tissue cells (4). Increasing evidence has indicated that the TME plays an important role in cancer development and metastasis $(4,5)$. Macrophages infiltrated in the TME are called tumor-associated macrophages (TAMs) and are key orchestrators in TME $(6,7)$. TAMs play a critical role in the regulation of tumor growth and progression $(8,9)$. The high density of TAMs is correlated with a poor prognosis in various types of cancer including GC (9-15). However, the role of TAMs in GC and the underlying mechanism remain elusive.

Epithelial-mesenchymal transition (EMT) is a process by which epithelial cells lose their epithelial attributes and acquire a mesenchymal cell phenotype, which is a key process in promoting tumor cell metastasis (16). TAMs induce EMT in pancreatic and non-small cell lung cancer (NSCLC), breast cancer, cholangiocarcinoma and hepatocellular carcinoma (17-22). Using literature review, we highlighted the role of TAMs in the regulation of EMT during tumorigenesis (23). In GC, it has been reported that infiltration of TAMs is associated with EMT-related proteins in human GC tissues, but the exact mechanism has not been clarified (14). 
In the present study, we aimed to investigate the role of TAMs in GC through the regulation of EMT. FOXQ1, a forkhead box-containing transcription factor, promotes EMT and metastasis in various types of cancer including GC (24-33). The potential involvement of FOXQ1 in TAM-induced EMT and metastasis was also investigated.

\section{Materials and methods}

Cell culture, reagent and plasmid. The THP-1 cell line and the human GC cell lines, MKN45 and MKN74, were used in the present study. All cell lines were obtained from the Cell Bank of Shanghai (Shanghai, China). The cells were grown in RPMI-1640 medium (Gibco, Gaithersburg, MD, USA) that was supplemented with $10 \%$ fetal bovine serum (FBS), penicillin $(100 \mathrm{U} / \mathrm{ml})$ and streptomycin $(100 \mathrm{mg} / \mathrm{ml})$, and were incubated in a humidified atmosphere containing $5 \% \mathrm{CO}_{2}$ at $37^{\circ} \mathrm{C}$ and the medium was replaced three times/week.

Rabbit anti-FOXQ1 (1:100; ab51340; Abcam, Cambridge, MA, USA), rabbit anti-E-cadherin (1:100; AF0131; Affinity, Sterling, VA, USA), rabbit anti-vimentin (1:100; 5741; Cell Signaling Technology, Inc., Beverly, MA, USA) and mouse anti- $\beta$-actin (1:100; T0022; Affinity) were used as primary antibodies. The FOXQ1 shRNA lentiviral particle containing FOXQ1 shRNA sequences was purchased from Santa Cruz Biotechnology, Inc. (Santa Cruz, CA, USA). MKN45 and MKN74 cells were infected with shFOXQ1 lentiviral particles and a negative control for $48 \mathrm{~h}$ and followed by $2 \mathrm{mg} / \mathrm{ml}$ of puromycin selection.

Co-culture of GC cells and macrophage. GC MKN45 and MKN74 cells $\left(10^{5}\right.$ cells/well) were seeded into 24 -well plates (BD Biosciences, Franklin Lakes, NJ, USA) in RPMI-1640 medium supplemented with $10 \%$ FBS, penicillin and streptomycin. THP-1 cells ( $3 \times 10^{5}$ cells/insert) were seeded into the upper chamber of a Transwell insert with a pore size of $8.0-\mu \mathrm{m}$ (Corning Incorporated, Kennebunk, ME, USA) in RPMI-1640 medium supplemented with $10 \%$ FBS, penicillin and streptomycin. The cells were incubated in a humidified atmosphere containing $5 \% \mathrm{CO}_{2}$ at $37^{\circ} \mathrm{C}$.

Quantitative real-time reverse transcription PCR. Total RNA was extracted with TRIzol reagent (Invitrogen, Carlsbad, CA, USA) according to the manufacturer's instructions and reverse-transcribed into cDNA using a reverse transcription kit from Takara Biotechnology, Ltd. (Dalian, China). After adjusting the cDNA concentration in all groups, qRT-PCR was performed using the CFX96 Real-Time PCR Detection System (Bio-Rad, Hercules, CA, USA) with SYBR-Green. The PCR conditions were as follows: pre-denaturation at $95^{\circ} \mathrm{C}$ for $30 \mathrm{sec} ; 35$ cycles of denaturation $\left(95^{\circ} \mathrm{C}\right.$ for $\left.5 \mathrm{sec}\right)$, annealing $\left(55-60^{\circ} \mathrm{C}\right.$ for $\left.30 \mathrm{sec}\right)$ and extension $\left(72^{\circ} \mathrm{C}\right.$ for $\left.1 \mathrm{~min}\right)$; and a final extension at $72^{\circ} \mathrm{C}$ for $10 \mathrm{~min}$. The relative level of gene expression was calculated using the $\Delta \Delta \mathrm{Ct}$ method with normalization to GAPDH. All experiments were performed in triplicate. The primers used are listed in Table I.

Western blotting. Protein expression levels were analyzed by western blotting standard protocols. The protein of MKN45 and MKN74 cells was extracted using RIPA lysis buffer
Table I. Primer sequences for qRT-PCR amplification of different genes.

\begin{tabular}{lll}
\hline Gene & & \multicolumn{2}{c}{ Primer } \\
\hline GAPDH & F & 5'-CTTTGGTATCGTGGAAGGACTC-3' \\
& R & 5'-GTAGAGGCAGGGATGATGTTCT-3' \\
E-cadherin & F & 5'-TGGCTTCCCTCTTTCATCTCC-3' \\
& R & 5'-TCATAGTTCCGCTCTGTCTTTGG-3' \\
Vimentin & F & 5'-TCAATGTTAAGATGGCCCTTG-3' \\
& R & 5'-TGAGTGGGTATCAACCAGAGG-3' \\
FOXQ1 & F & 5'-TGATTTCTTGCTATTGACCGATGC-3' \\
& R & 5'-GCCCAAGGAGACCACAGTTAGAG-3'
\end{tabular}

F, forward; R, reverse.

(Beyotime, Haimen, China) with protease inhibitor PMSF (CWBiotech, Beijing, China) according to the manufacturer's instructions. Briefly, $20 \mu \mathrm{l}$ of total protein extracts was resolved by denaturing sodium dodecyl sulfate-polyacrylamide gel electrophoresis and transferred to polyvinylidene difluoride membranes. The membranes were blocked with 5\% non-fat milk, and then incubated with primary antibodies. Then, the blots were washed and probed with the respective secondary peroxidase-conjugated antibodies. Signals were detected using a chemiluminescence solvent (Thermo Scientific, Rockford, IL, USA).

Transwell invasion assay. THP- 1 cells $\left(10^{5}\right.$ cells/well) were seeded in 24-well plates in RPMI-1640 medium supplemented with $10 \%$ FBS. MKN45 and MKN74 cells ( $10^{4}$ cells/insert) were seeded into the upper chamber of a Transwell insert with a pore size of $8.0 \mu \mathrm{m}$ precoated to a Matrigel Basement Membrane Matrix (BD Biosciences) in RPMI-1640 medium supplemented with $1 \%$ FBS. After incubated in a humidified atmosphere containing $5 \% \mathrm{CO}_{2}$ at $37^{\circ} \mathrm{C}$ for $24 \mathrm{~h}$, the cells migrated to the lower surface of the membranes were fixed with $4 \%$ paraformaldehyde, stained with $0.1 \%$ crystal violet and counted under a microscope. All these samples were plated three times.

Wound healing assay. Approximately $5 \times 10^{4} \mathrm{GC}$ cells from different groups were seeded in 24 -well plates and incubated for $24 \mathrm{~h}$. Then, the monolayer cells were disrupted by scratching with a $10 \mu 1$ microsterile pipette tips. Images were captured at 0 and $24 \mathrm{~h}$ in a phase-contract microscope. The assays were performed in triplicate, and four fields of each well were assessed.

Tissue specimens, immunohistochemistry and assessment of CD68 and FOXQ1 expression. All specimens were obtained from the Department of Surgical Oncology, The First Affiliated Hospital, Xi'an Jiaotong University and the Department of Surgical Oncology, the 215th Hospital of Shaanxi Province. Detailed information on the specimens was previously provided (14). The present study was approved by the Protection of Human Subjects Committee of the First 
A
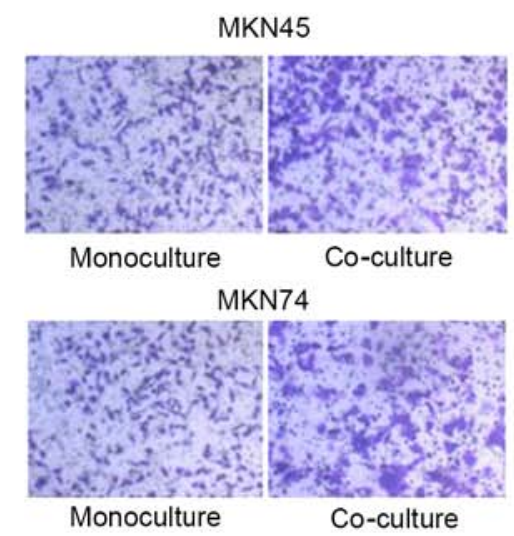

C

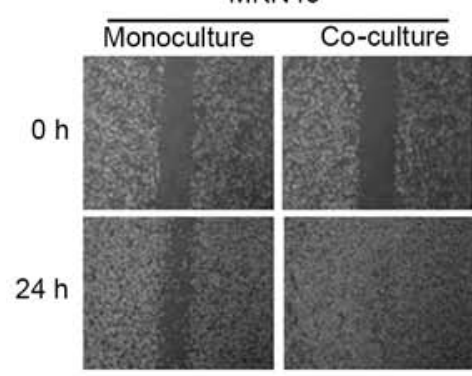

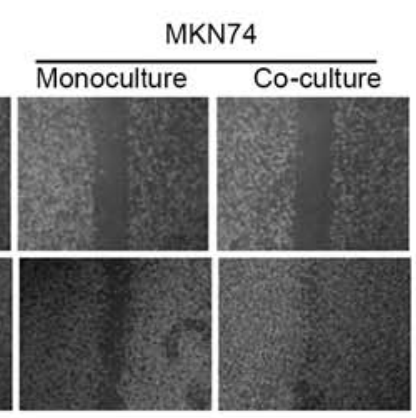

B

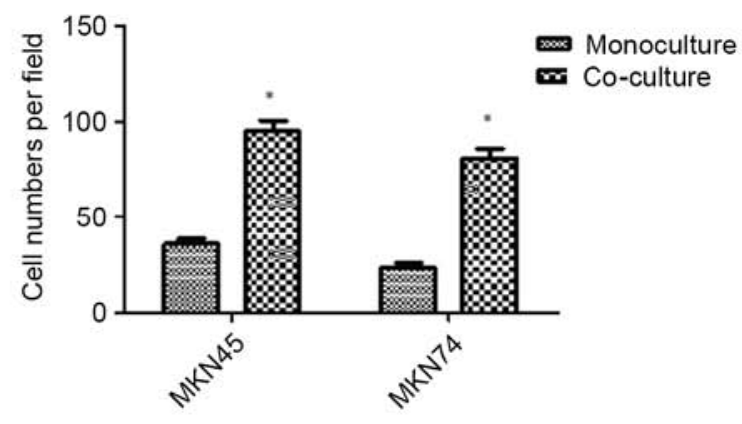

D

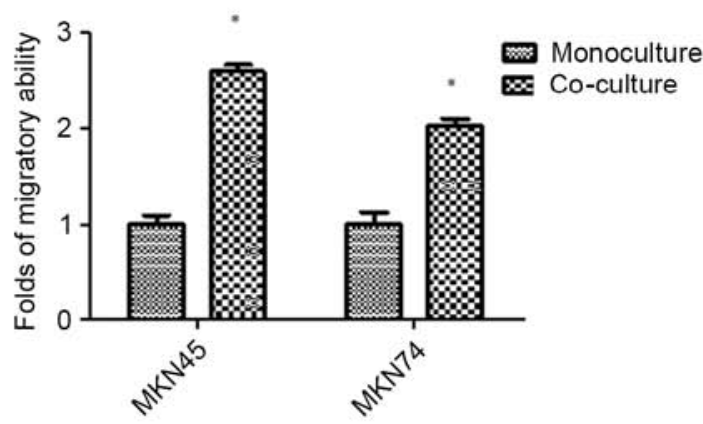

Figure 1. Co-culture with TAMs promotes invasion and migration in GC cells. (A and B) The invasion ability of GC cells co-cultured with TAMs and its control was assessed by Transwell assay. (C and D) The migration ability of GC cells co-cultured with TAMs and its control was assessed by wound-healing assay. Values are depicted as the mean $\pm \mathrm{SD} ;{ }^{*} \mathrm{P}<0.05$. GC, gastric cancer; TAMs, tumor-associated macrophages.

Affiliated Hospital, Xi'an Jiaotong University and complied with the Helsinki Declaration. The tissues specimens were fixed in neutral buffered formalin and embedded in paraffin wax. The sections of 4-mm thickness were cut and mounted on charged glass slides. Antigen retrieval was performed using citrate buffer at $\mathrm{pH}$ 6.0. Immunohistochemical staining was performed using mouse anti-human CD68 (1:100; ZM-0060; Beijing Zhongshan Biotechnology, Beijing, China) and rabbit anti-human FOXQ1 (1:100; bs-16175R; Beijing Bioss Biotechnology, Beijing, China). The streptavidin-peroxidase technique (SP-9001 Golden Bridge Int., Beijing, China) was used. An irrelevant rabbit antiserum served as a negative control. The sections were stained with $0.02 \%$ diaminobenzidine (DAB) solution followed by counterstaining with hematoxylin. Staining results were classified into high expression and low expression as previously described $(14,32)$.

Statistical analysis. Data analyses were performed using SPSS statistical package 16.0 (SPSS Institute, Chicago, IL, USA) or Prism (GraphPad Software, Inc., La Jolla, CA, USA). A P-value $<0.05$ was considered to indicate a statistically significant result. The $\chi^{2}$ test was used to analyze the correlation between CD68 and FOXQ1 expression.

\section{Results}

Co-culture with TAMs promotes invasion and migration in GC cells. After 24 h of THP-1 co-culture, GC (MKN45 and MKN74) cells were subjected to Transwell invasion assays. As shown in Fig. 1A and B, THP-1 co-culture resulted in an increase in the invasive ability of both MKN45 and MKN74 cells. The result was confirmed by wound-healing assay. Compared with the control, GC cells co-cultured with THP-1 cells exhibited a faster closure of the wound (Fig. 1C and D).

Co-culture with TAMs induces EMT in GC cells. Western blotting and RT-PCR were used to analyze the EMT markers in MKN45 and MKN74 cells after being co-cultured with THP-1. As shown in Fig. 2, the expression of epithelial marker E-cadherin was downregulated, while the mesenchymal marker vimentin was upregulated.

FOXQ1 is involved in the EMT of GC cells induced by co-culture with TAMs. A previous study revealed that FOXQ1 promoted EMT and metastasis in GC (32). The potential involvement of FOXQ1 in TAM-induced EMT and metastasis is still unknown. In the present study, MKN45 and MKN74 cells were co-cultured with THP-1. The results revealed that co-culture with THP-1 significantly increased the expression of FOXQ1 at both the mRNA and protein levels of MKN45 and MKN74 cells (Fig. 3A and B). Thereafter, transfection of shRNA targeting FOXQ1 resulted in pronounced knockdown of mRNA and protein levels in MKN45 and MKN74 cells (Fig. 3C and D).

FOXQ1 is essential for TAM-induced EMT and metastasis in $G C$ cells. To confirm the role of FOXQ1 in TAM-induced EMT and invasion in GC cells, we silenced FOXQ1 expression in MKN45 and MKN74 cells with shRNA before being co-cultured with THP-1 cells. As shown in Fig. 4, the 


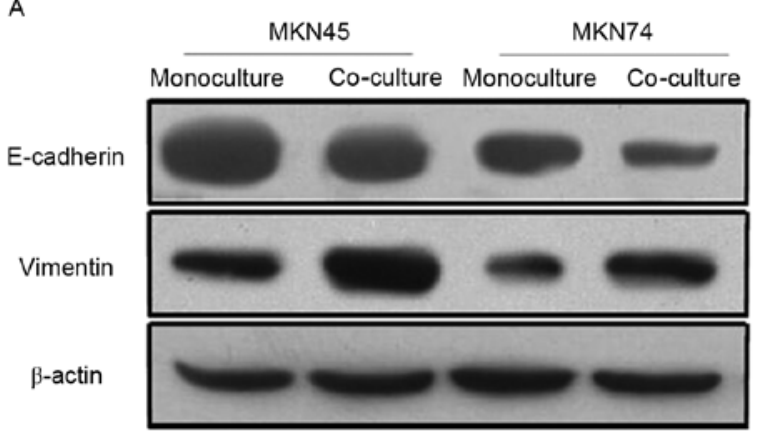

C

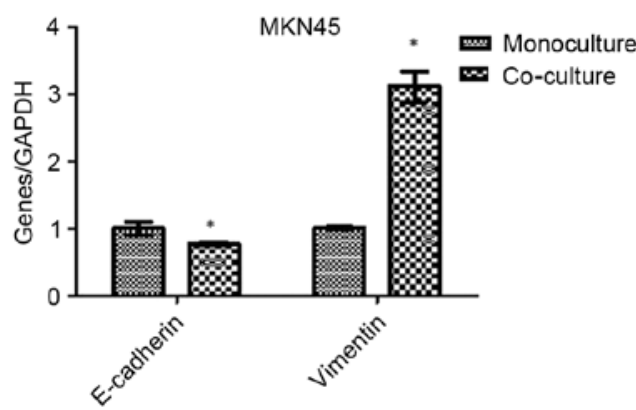

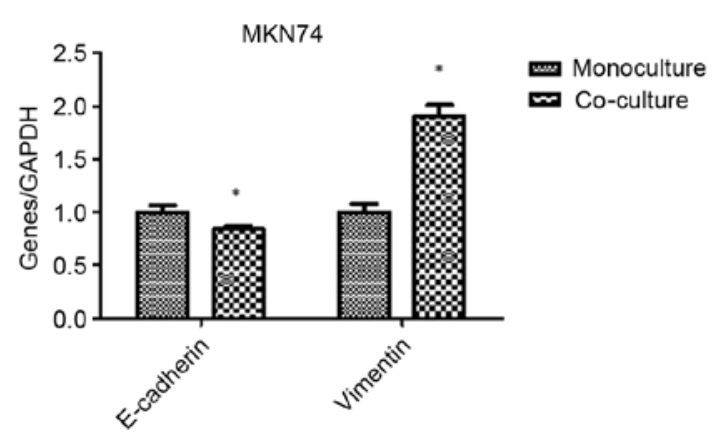

Figure 2. Co-culture with TAMs induces EMT in GC cells. (A) The EMT markers in MKN45 and MKN74 cells were analyzed using western blotting after being co-cultured with THP-1 cells. (B and C) The EMT markers in MKN45 and MKN74 cells were analyzed by RT-PCR after being co-cultured with THP-1 cells; "P<0.05. TAMs, tumor-associated macrophages; EMT, epithelial-mesenchymal transition; GC, gastric cancer.

A

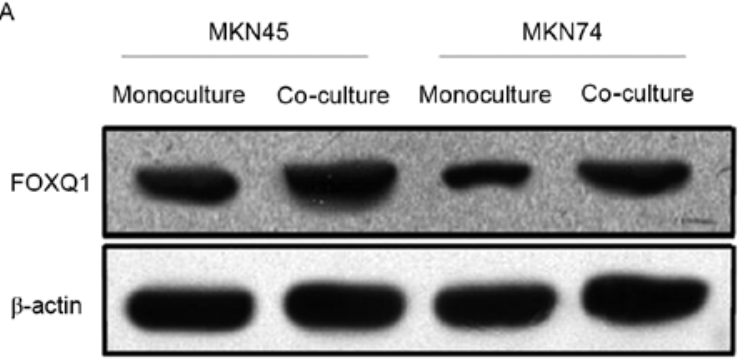

C

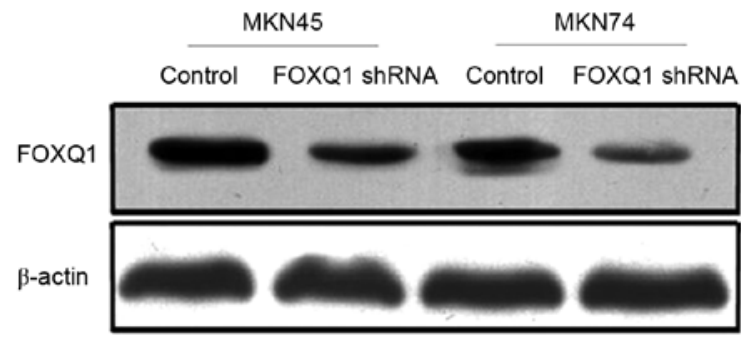

B

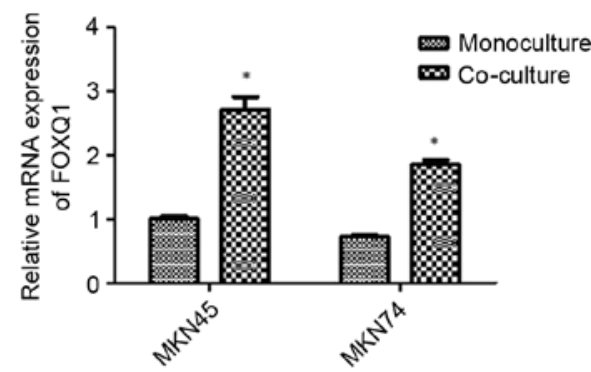

D

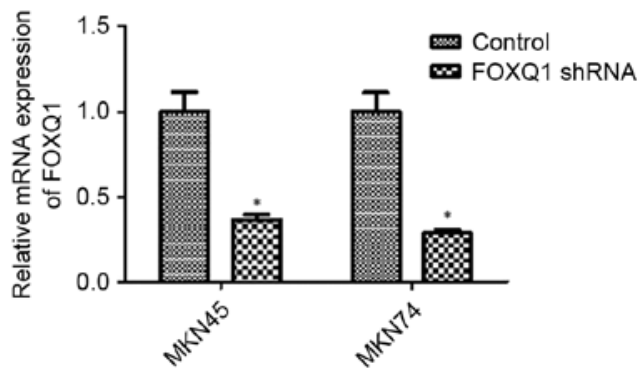

Figure 3. FOXQ1 is involved in EMT of GC cells induced by co-culture with TAMs. (A and B) Co-culture with THP-1 cells significantly increased the expression of FOXQ1 at both the mRNA and protein levels of MKN45 and MKN74 cells. (C and D) Transfection with shRNA targeting FOXQ1 resulted in the pronounced knockdown of mRNA and protein levels in MKN45 and MKN74 cells. Values are depicted as the mean \pm SD; ${ }^{*} \mathrm{P}<0.05$. FOXQ1, forkhead box Q1; EMT, epithelial-mesenchymal transition; GC, gastric cancer; TAMs, tumor-associated macrophages.

expression of mesenchymal marker vimentin was decreased, while the epithelial marker E-cadherin was increased in both MKN45-FOXQ1-shRNA and MKN74-FOXQ1-shRNA cells co-cultured with THP-1 cells. Transwell invasion assays revealed that silencing of FOXQ1 in MKN45 and MKN74 cells decreased their invasive ability after being co-cultured with THP-1 (Fig. 5A and B). Wound-healing assay indicated that silencing of FOXQ1 in GC MKN45 and MKN74 cells co-cultured with THP-1 cells exhibited a slower closure of the wound (Fig. 5C and D). These results clearly revealed that silencing of FOXQ1 blocked the effect of TAM-enhanced EMT and metastasis of GC cells. 
A

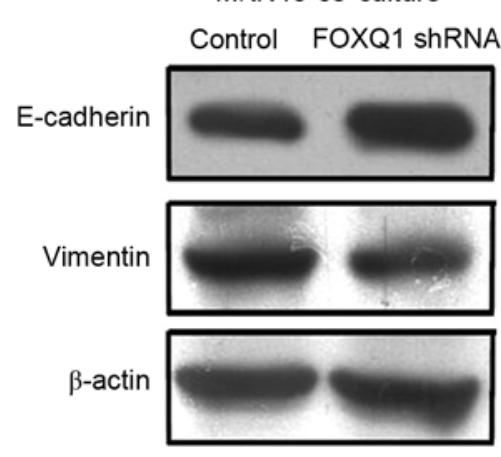

C

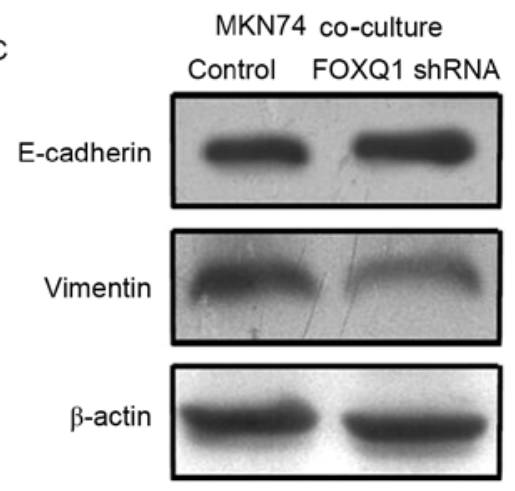

B

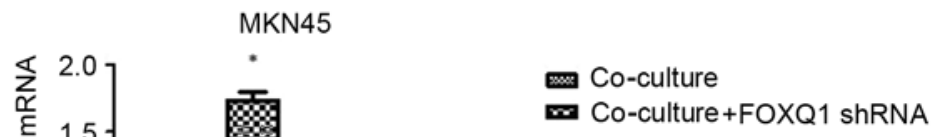

Figure 4. FOXQ1 is essential for TAM-induced EMT in GC cells. (A and B) The expression of mesenchymal marker vimentin was decreased, while the epithelial marker E-cadherin was increased in MKN45-FOXQ1-shRNA cells co-cultured with THP-1 cells. (C and D) The expression of mesenchymal marker vimentin was decreased, while the epithelial marker E-cadherin was increased in MKN74-FOXQ1-shRNA cells co-cultured with THP-1 cells. Values are depicted as the mean $\pm \mathrm{SD}$; "P<0.05. FOXQ1, forkhead box Q1; TAM, tumor-associated macrophage; EMT, epithelial-mesenchymal transition; GC, gastric cancer.

A

C

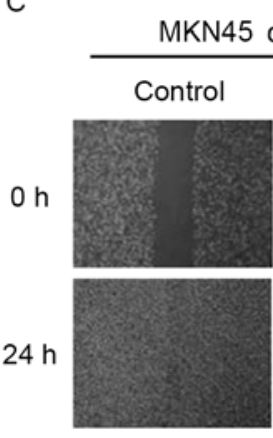

MKN45 co-culture

1 ShRNA

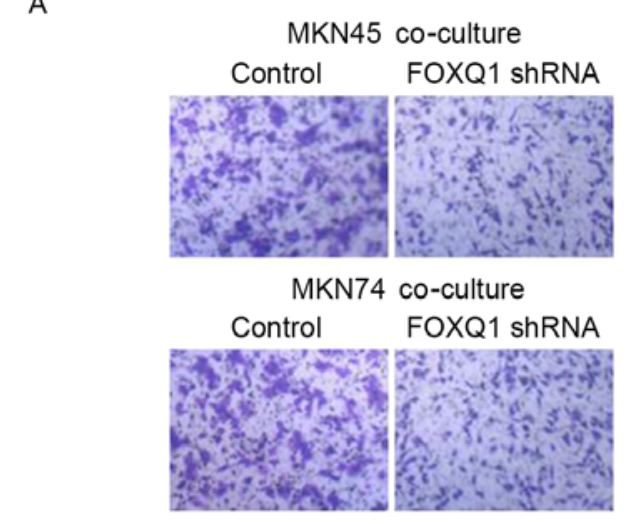

D

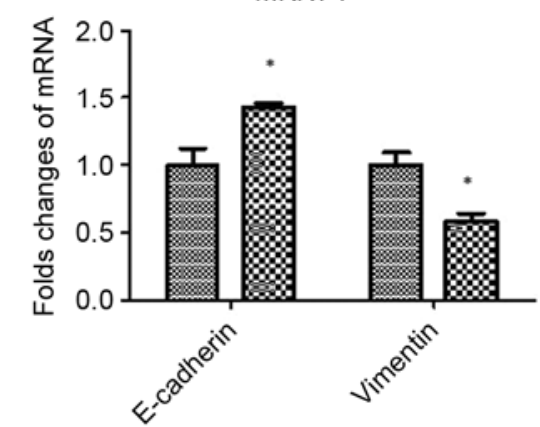

Co-culture

Co-culture+FOXQ1 shRNA
B

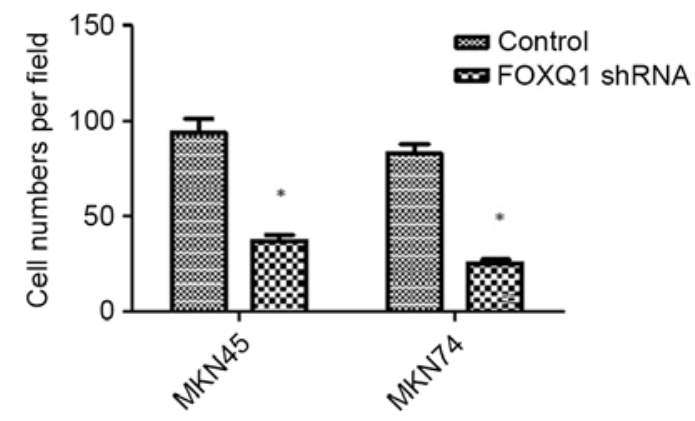

D

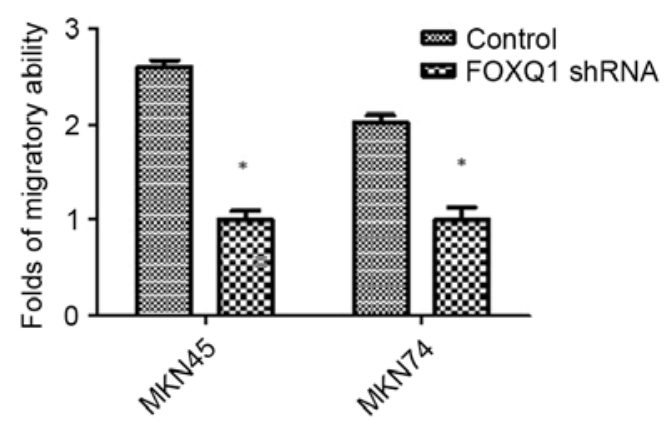

Figure 5. FOXQ1 is essential for TAM-induced metastasis in GC cells. (A and B) Transwell invasion assays revealed that silencing of FOXQ1 in MKN45 and MKN74 cells decreased their invasive ability after being co-cultured with THP-1 cells. (C and D) Wound-healing assay indicated that silencing of FOXQ1 in GC MKN45 and MKN74 cells co-cultured with THP-1 cells exhibited a slower closure of the wound. Values are depicted as the mean \pm SD; "P<0.05. FOXQ1, forkhead box Q1; TAM, tumor-associated macrophage; GC, gastric cancer. 
A

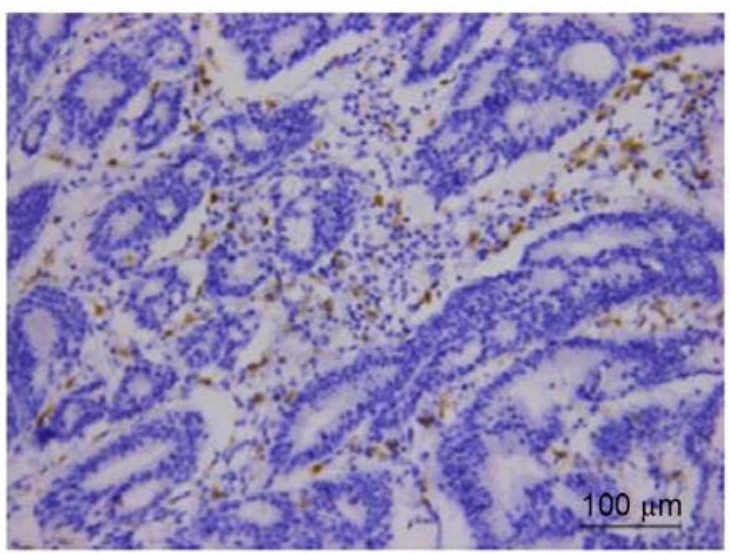

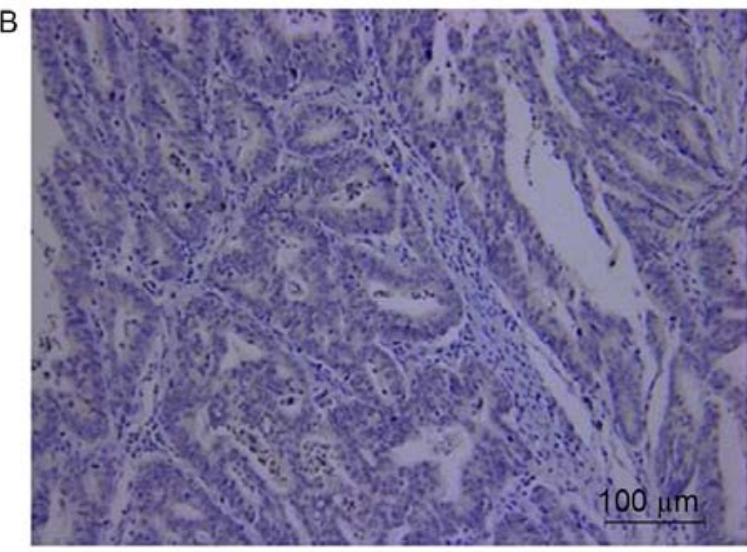

Figure 6. Correlation of TAM marker CD68 and FOXQ1 expression in GC tissues. High expression of (A) CD68 was correlated with anomalous positivity of (B) FOXQ1 expression. TAM, tumor-associated macrophage; FOXQ1, forkhead box Q1; GC, gastric cancer.

Table II. The association between CD68 and FOXQ1.

\begin{tabular}{lllll}
\hline & \multicolumn{2}{c}{ CD68 } & & \\
\cline { 2 - 3 } & High & Low & r & P-value \\
\hline FOXQ1 & & & & \\
High & 79 & 24 & 0.613 & 0.000 \\
Low & 11 & 64 & & \\
\hline
\end{tabular}

Correlation of TAM marker CD68 and FOXQ1 expression in $G C$ tissues. Respective photomicrographs of immunohistochemical staining of CD68 and FOXQ1 are shown in Fig. 6. The relationship between the expression of CD68 and FOXQ1 was calculated and has been outlined in Table II. The result revealed that high expression of CD68 was correlated with positive FOXQ1 expression $(r=0.613 ; \mathrm{P}<0.001)$ in clinical GC samples.

\section{Discussion}

The tumor microenvironment (TME) is comprised of macrophages, fibroblasts, lymphocytes, endothelial cells, adipocytes, perivascular cells, neurons and extracellular matrix (ECM) components, and plays an important role in cancer invasion and metastasis (4). TAMs are key orchestrators and a set of macrophages of the TME $(6,7)$. TAMs play a critical role in regulating tumor growth and progression $(8,9)$. The high density of TAMs is correlated with a poor prognosis in various types of cancer including gastric cancer (GC) (9-15). However, the role of TAMs in GC and the underlying mechanism remain elusive.

In the present study, we used THP-1 cells as a substitute to investigate the impact of TAMs on GC cells. Transwell invasion and wound healing assays indicated that co-cultured GC cells with TAMs significantly promoted GC cell invasion and migration. Loss of epithelial marker E-cadherin expression and gain of mesenchymal marker vimentin expression is a major hallmark of EMT (34). Aberrant reactivation of EMT promoted tumor cell migration and invasion (35). In the present study, E-cadherin expression in GC cells co-cultured with TAMs was decreased, while vimentin expression in GC cells co-cultured with TAMs was increased. This indicated that GC cells co-cultured with TAMs underwent EMT. These results revealed that TAMs promoted GC cell invasion and migration through EMT.

FOXQ1 is a member of the large forkhead (FOX) transcription factor family (36). It is expressed in different tissues and plays an important role in development, metabolism, aging and cancer (37-39). It is an $\sim 42 \mathrm{kDa}$ protein initially shown to be involved in hair follicle differentiation, gastrulation and mucin production in mice (40-42). Several recent studies demonstrated that increased FOXQ1 expression in many human types of cancer, including esophageal, breast and colorectal cancer, hepatocellular carcinoma and non-small cell lung cancer, was correlated with metastasis and poor prognosis $(25-27,29,30,43-48)$. FOXQ1 has been shown to be involved in the regulation of EMT (37). In breast cancer and non-small cell lung cancer, FOXQ1 has been shown to promote EMT by regulating the expression of E-cadherin, $\beta$-catenin and vimentin $(26,27,30)$. In hepatocellular carcinoma, FOXQ1 was reported to induce EMT and enhance the invasive capability by activating transcription ZEB2 and Versican V1 (45). In bladder cancer, knockdown of FOXQ1 inhibited invasion and metastasis via the reversal of EMT (28). These studies indicated that FOXQ1 plays an important role in EMT, and subsequently in the invasion and metastasis of many types of cancer. A recent study reported that FOXQ1 promoted GC metastasis through upregulation of Snail (32).

We hypothesized that TAMs promote GC cell EMT, invasion and migration through the FOXQ1 pathway. RT-PCR and western blot results revealed that co-culture with TAMs significantly increased FOXQ1 expression at both the mRNA and protein levels of GC cells, which indicated that FOXQ1 was involved in the EMT of GC cells induced by TAMs. Then, FOXQ1 shRNA was used to silence the expression of FOXQ1. The results revealed that promotion of TAMs in EMT was suppressed. Meanwhile, the promotion of TAMs on the abilities of migration and invasion in GC cells were also inhibited.

Furthermore, we investigated the correlation of TAM marker CD68 and FOXQ1 expression in GC tissues. In accordance with the present in vitro study, the result revealed that high expression of CD68 was correlated with positive FOXQ1 expression $(\mathrm{r}=0.613 ; \mathrm{P}<0.001)$ in clinical $\mathrm{GC}$ samples. 
In conclusion, our data provided evidence that TAMs promote EMT, invasion and migration of GC cells via FOXQ1. Therefore, the TAM/FOXQ1 axis may represent a novel target for GC cells.

\section{Acknowledgements}

The present study was supported by the National Natural Science Foundation of China (no. 81301981), and the Fundamental Research Funds for the Central Universities.

\section{References}

1. Torre LA, Bray F, Siegel RL, Ferlay J, Lortet-Tieulent J and Jemal A: Global cancer statistics, 2012. CA Cancer J Clin 65 : 87-108, 2015.

2. DeSantis CE, Lin CC, Mariotto AB, Siegel RL, Stein KD, Kramer JL, Alteri R, Robbins AS and Jemal A: Cancer treatment and survivorship statistics, 2014. CA Cancer J Clin 64: 252-271, 2014.

3. Zeng H, Zheng R, Guo Y, Zhang S, Zou X, Wang N, Zhang L, Tang J, Chen J, Wei K, et al: Cancer survival in China, 2003-2005: A population-based study. Int J Cancer 136: 1921-1930, 2015.

4. Whiteside TL: The tumor microenvironment and its role in promoting tumor growth. Oncogene 27: 5904-5912, 2008.

5. Lee CH, Liu SY, Chou KC, Yeh CT, Shiah SG, Huang RY, Cheng JC, Yen CY and Shieh YS: Tumor-associated macrophages promote oral cancer progression through activation of the Axl signaling pathway. Ann Surg Oncol 21: 1031-1037, 2014.

6. Quail DF and Joyce JA: Microenvironmental regulation of tumor progression and metastasis. Nat Med 19: 1423-1437, 2013.

7. Joyce JA and Pollard JW: Microenvironmental regulation of metastasis. Nat Rev Cancer 9: 239-252, 2009.

8. Fan QM, Jing YY, Yu GF, Kou XR, Ye F, Gao L, Li R, Zhao QD, Yang Y, Lu ZH, et al: Tumor-associated macrophages promote cancer stem cell-like properties via transforming growth factor-beta1-induced epithelial-mesenchymal transition in hepatocellular carcinoma. Cancer Lett 352: 160-168, 2014.

9. Hu Y, He MY, Zhu LF, Yang CC, Zhou ML, Wang Q, Zhang W, Zheng YY, Wang DM, Xu ZQ, et al: Tumor-associated macrophages correlate with the clinicopathological features and poor outcomes via inducing epithelial to mesenchymal transition in oral squamous cell carcinoma. J Exp Clin Cancer Res 35: 12, 2016.

10. Zhang BC, Gao J, Wang J, Rao ZG, Wang BC and Gao JF: Tumor-associated macrophages infiltration is associated with peritumoral lymphangiogenesis and poor prognosis in lung adenocarcinoma. Med Oncol 28: 1447-1452, 2011.

11. Suriano F, Santini D, Perrone G, Amato M, Vincenzi B, Tonini G, Muda A, Boggia S, Buscarini M and Pantano F: Tumor associated macrophages polarization dictates the efficacy of BCG instillation in non-muscle invasive urothelial bladder cancer. J Exp Clin Cancer Res 32: 87, 2013.

12. Yan Y, Zhang J, Li JH, Liu X, Wang JZ, Qu HY, Wang JS and Duan XY: High tumor-associated macrophages infiltration is associated with poor prognosis and may contribute to the phenomenon of epithelial-mesenchymal transition in gastric cancer. Onco Targets Ther 9: 3975-3983, 2016.

13. Mei J, Xiao Z, Guo C, Pu Q, Ma L, Liu C, Lin F, Liao H, You Z and Liu L: Prognostic impact of tumor-associated macrophage infiltration in non-small cell lung cancer: A systemic review and meta-analysis. Oncotarget 7: 34217-34228, 2016.

14. Zhang J, Yan Y, Yang Y, Wang L, Li M, Wang J, Liu X, Duan X and Wang J: High infiltration of tumor-associated macrophages influences poor prognosis in human gastric cancer patients, associates with the phenomenon of EMT. Medicine 95: e2636, 2016.

15. Zhang H, Wang X, Shen Z, Xu J, Qin J and Sun Y: Infiltration of diametrically polarized macrophages predicts overall survival of patients with gastric cancer after surgical resection. Gastric Cancer 18: 740-750, 2015.

16. Thiery JP: Epithelial-mesenchymal transitions in tumour progression. Nat Rev Cancer 2: 442-454, 2002

17. Bonde AK, Tischler V, Kumar S, Soltermann A and Schwendener RA: Intratumoral macrophages contribute to epithelial-mesenchymal transition in solid tumors. BMC Cancer 12: 35, 2012.
18. Liu CY, Xu JY, Shi XY, Huang W, Ruan TY, Xie P and Ding JL: M2-polarized tumor-associated macrophages promoted epithelialmesenchymal transition in pancreatic cancer cells, partially through TLR4/IL-10 signaling pathway. Lab Invest 93: 844-854, 2013.

19. Ravi J, Elbaz M, Wani NA, Nasser MW and Ganju RK: Cannabinoid receptor-2 agonist inhibits macrophage induced EMT in non-small cell lung cancer by downregulation of EGFR pathway. Mol Carcinog 55: 2063-2076, 2016.

20. Deng YR, Liu WB, Lian ZX, Li X and Hou X: Sorafenib inhibits macrophage-mediated epithelial-mesenchymal transition in hepatocellular carcinoma. Oncotarget 7: 38292-38305, 2016.

21. Su S, Liu Q, Chen J, Chen J, Chen F, He C, Huang D, Wu W, Lin L, Huang W, et al: A positive feedback loop between mesenchymal-like cancer cells and macrophages is essential to breast cancer metastasis. Cancer Cell 25: 605-620, 2014.

22. Techasen A, Loilome W, Namwat N, Dokduang H, Jongthawin J and Yongvanit P: Cytokines released from activated human macrophages induce epithelial mesenchymal transition markers of cholangiocarcinoma cells. Asian Pac J Cancer Prev 13 (Suppl): $115-118,2012$.

23. Zhang J, Yao H, Song G, Liao X, Xian Y and Li W: Regulation of epithelial-mesenchymal transition by tumor-associated macrophages in cancer. Am J Transl Res 7: 1699-1711, 2015.

24. Zhang M, Xu Q, Yan S, Li Z, Yan W and Jia X: Suppression of forkhead box Q1 by microRNA-506 represses the proliferation and epithelial-mesenchymal transition of cervical cancer cells. Oncol Rep 35: 3106-3114, 2016.

25. Qiao Y, Jiang X, Lee ST, Karuturi RK, Hooi SC and Yu Q: FOXQ1 regulates epithelial-mesenchymal transition in human cancers. Cancer Res 71: 3076-3086, 2011.

26. Zhang H, Meng F, Liu G, Zhang B, Zhu J, Wu F, Ethier SP, Miller $\mathrm{F}$ and Wu G: Forkhead transcription factor foxql promotes epithelial-mesenchymal transition and breast cancer metastasis. Cancer Res 71: 1292-1301, 2011.

27. Feng J, Zhang X, Zhu H, Wang X, Ni S and Huang J: FoxQ1 overexpression influences poor prognosis in non-small cell lung cancer, associates with the phenomenon of EMT. PLoS One 7: e39937, 2012

28. Zhu Z, Zhu Z, Pang Z, Xing Y, Wan F, Lan D and Wang H: Short hairpin RNA targeting FOXQ1 inhibits invasion and metastasis via the reversal of epithelial-mesenchymal transition in bladder cancer. Int J Oncol 42: 1271-1278, 2013.

29. Sehrawat A, Kim SH, Vogt A and Singh SV: Suppression of FOXQ1 in benzyl isothiocyanate-mediated inhibition of epithelial-mesenchymal transition in human breast cancer cells. Carcinogenesis 34: 864-873, 2013.

30. Feng J, Xu L, Ni S, Gu J, Zhu H, Wang H, Zhang S, Zhang W and Huang J: Involvement of FoxQ1 in NSCLC through regulating EMT and increasing chemosensitivity. Oncotarget 5: 9689-9702, 2014.

31. Fan DM, Feng XS, Qi PW and Chen YW: Forkhead factor FOXQ1 promotes TGF- $\beta 1$ expression and induces epithelialmesenchymal transition. Mol Cell Biochem 397: 179-186, 2014.

32. Zhang J, Liu Y, Zhang J, Cui X, Li G, Wang J, Ren H and Zhang Y: FOXQ1 promotes gastric cancer metastasis through upregulation of Snail. Oncol Rep 35: 3607-3613, 2016.

33. Xiang XJ, Deng J, Liu YW, Wan LY, Feng M, Chen J and Xiong JP: MiR-1271 inhibits cell proliferation, invasion and EMT in gastric cancer by targeting FOXQ1. Cell Physiol Biochem 36: 1382-1394, 2015 .

34. Thiery JP, Acloque H, Huang RY and Nieto MA: Epithelialmesenchymal transitions in development and disease. Cell 139: 871-890, 2009.

35. Thiery JP and Sleeman JP: Complex networks orchestrate epithelial-mesenchymal transitions. Nat Rev Mol Cell Biol 7: 131-142, 2006.

36. Myatt SS and Lam EW: The emerging roles of forkhead box (Fox) proteins in cancer. Nat Rev Cancer 7: 847-859, 2007.

37. Feuerborn A, Srivastava PK, Küffer S, Grandy WA, Sijmonsma TP, Gretz N, Brors B and Gröne HJ: The Forkhead factor FoxQ1 influences epithelial differentiation. J Cell Physiol 226: 710-719, 2011.

38. Eijkelenboom A and Burgering BM: FOXOs: Signalling integrators for homeostasis maintenance. Nat Rev Mol Cell Biol 14: 83-97, 2013.

39. Jonsson $\mathrm{H}$ and Peng SL: Forkhead transcription factors in immunology. Cell Mol Life Sci 62: 397-409, 2005.

40. Goering W, Adham IM, Pasche B, Manner J, Ochs M, Engel W and Zoll B: Impairment of gastric acid secretion and increase of embryonic lethality in Foxq1-deficient mice. Cytogenet Genome Res 121: 88-95, 2008. 
41. Hong HK, Noveroske JK, Headon DJ, Liu T, Sy MS, Justice MJ and Chakravarti A: The winged helix/forkhead transcription factor Foxql regulates differentiation of hair in satin mice. Genesis 29: 163-171, 2001.

42. Potter CS, Peterson RL, Barth JL, Pruett ND, Jacobs DF, Kern MJ, Argraves WS, Sundberg JP and Awgulewitsch A: Evidence that the satin hair mutant gene Foxql is among multiple and functionally diverse regulatory targets for Hoxcl3 during hair follicle differentiation. J Biol Chem 281: 29245-29255, 2006.

43. Kaneda H, Arao T, Tanaka K, Tamura D, Aomatsu K, Kudo K, Sakai K, De Velasco MA, Matsumoto K, Fujita Y, et al: FOXQ1 is overexpressed in colorectal cancer and enhances tumorigenicity and tumor growth. Cancer Res 70: 2053-2063, 2010.

44. Wang W, He S, Ji J, Huang J, Zhang S and Zhang Y: The prognostic significance of FOXQ1 oncogene overexpression in human hepatocellular carcinoma. Pathol Res Pract 209: 353-358, 2013.
45. Xia L, Huang W, Tian D, Zhang L, Qi X, Chen Z, Shang X, Nie Y and Wu K: Forkhead box Q1 promotes hepatocellular carcinoma metastasis by transactivating ZEB2 and VersicanV1 expression. Hepatology 59: 958-973, 2014

46. Pei Y, Wang P, Liu H, He F and Ming L: FOXQ1 promotes esophageal cancer proliferation and metastasis by negatively modulating CDH1. Biomed Pharmacother 74: 89-94, 2015.

47. Peng X, Luo Z, Kang Q, Deng D, Wang Q, Peng H, Wang S and Wei Z: FOXQ1 mediates the crosstalk between TGF- $\beta$ and Wnt signaling pathways in the progression of colorectal cancer. Cancer Biol Ther 16: 1099-1109, 2015.

48. Liang SH, Yan XZ, Wang BL, Jin HF, Yao LP, Li YN, Chen M, Nie YZ, Wang X, Guo XG, et al: Increased expression of FOXQ1 is a prognostic marker for patients with gastric cancer. Tumour Biol 34: 2605-2609, 2013. 